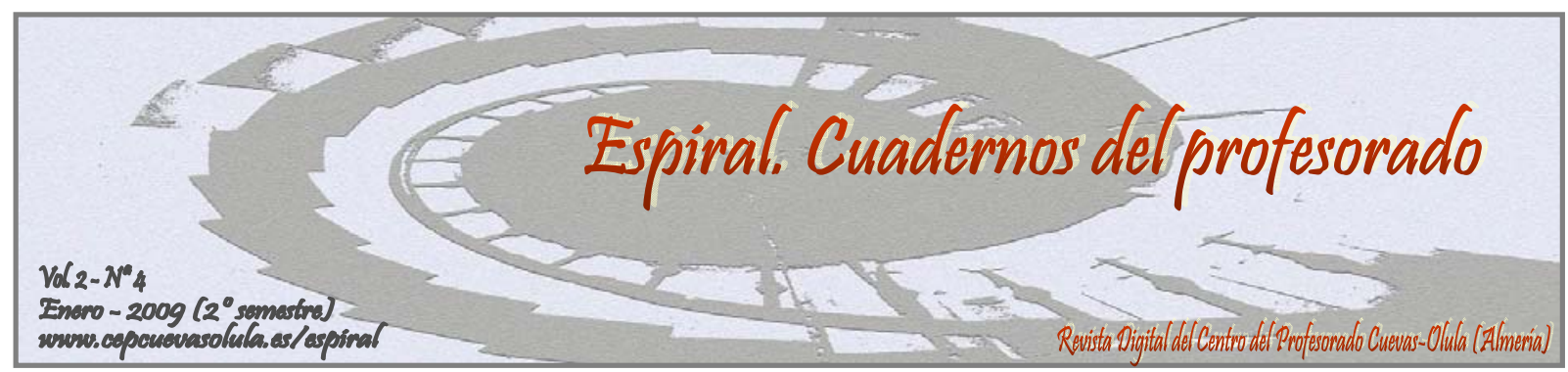

\title{
PRÁCTICAS DE SIMULACIÓN DE CORRESPONDENCIA COMERCIAL EN INGLÉS PARA ENTORNOS DE INGENIERÍA
}

\section{COMMERCIAL CORRESPONDENCE SIMULATION PRACTICE IN ENGLISH FOR ENGINEERING PURPOSES}

\author{
Luis Dochao Moreno ${ }^{(1)}$ y José Luis Llavona Arregui( ${ }^{(2)}$
}

\author{
(1) Escuela Técnica Superior de Ingenieros Aeronáuticos. Universidad Politécnica de Madrid

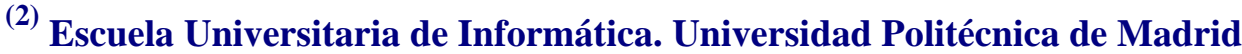

\begin{abstract}
RESUMEN: La práctica de correspondencia comercial y profesional en inglés para entornos de ingeniería se diseñó y aplicó a partir del curso 95/96 para los alumnos de las Escuelas: Universitaria de Informática y Técnica Superior de Ingenieros Aeronáuticos de la Universidad Politécnica de Madrid. Esta práctica se ideó con el principal objetivo de satisfacer ciertas necesidades por parte de los futuros ingenieros en cuyo programa de estudios durante la carrera existe una carencia de situaciones de simulación de las diferentes destrezas comunicativas. De todos es sabida la importancia que tiene la comunicación en nuestra sociedad, y los ingenieros recién incorporados al mundo laboral no son ajenos a esta necesidad. Con el fin de paliar parte de este espectro de necesidades, los profesores que llevamos a cabo la práctica brindamos esta posibilidad a los alumnos de las dos Escuelas ya mencionadas. La práctica consta de varias fases, cada una de las cuales se corresponde con una diferente función comunicativa. Gracias a esta práctica hemos ayudado a subsanar los errores más frecuentes que cometen los estudiantes al producir correspondencia comercial en inglés. En cuanto a la consecución de los objetivos, tenemos la palabra de algunos antiguos alumnos que afirman que la práctica les ha ayudado a desenvolverse en este campo con mayor facilidad que si no la hubieran realizado.
\end{abstract}

Palabras clave: práctica, ingeniería, inglés, aprendizaje, función comunicativa, correspondencia comercial.

ABSTRACT: The practice of commercial and professional correspondence in English for Engineering Purposes was designed and applied at the Polytechnic University of Madrid the academic year 1995/6. It was developed for the students of the Computer Science and the Aeronautical Engineering School students in order to mitigate the lack of simulation practices offered to them in their syllabuses. We are all aware of the importance of the communicative skills in today's society. Our students are not an exception and we decided to carry out this practice for this main purpose. This practice is divided into several parts, each corresponding to a different communicative skill. Since the beginning of this practice we have been aware of the common mistakes made by the students. Every academic year, our students have had the chance to get to know about these mistakes in order not to repeat them and approach correctness. Some former students have considered this practice to be of great help in their jobs, something they appreciate since it would have been harder for them in case they would not have had this opportunity.

Key words: practice, engineering, English, learning, communicative skills, commercial letter writing. 
Dochao, L. \& Llavona, J. L. (2009). Prácticas de simulación de correspondencia comercial en inglés para entronos de ingeniería [en línea]. Espiral. Cuadernos del Profesorado, 2(4), 34-42. Disponible en: http://www.cepcuevasolula.es/espiral.

Fecha de recepción: 22/03/2009

Fecha de aceptación: 05/05/2009
Enviar correspondencia a: Luis.dochao@upm.es

\section{1.- INTRODUCCIÓN.}

Este artículo surge a raíz de la experiencia de los profesores que han mantenido esta práctica desde el curso 95/96. Desde sus comienzos se ha pretendido que sea al tiempo un resumen de la labor realizada y un método de trabajo para aquel alumnado que careciera de referentes y experiencia prácticos para la redacción de textos en inglés para entornos de ingeniería. En cuanto al número de estudiantes participantes, la Escuela Universitaria de Informática ha venido realizando esta práctica con un número de 10 a 15 alumnos y alumnas para las asignaturas de: Inglés Técnico II e Inglés para Entornos Profesionales. Ambas en actividad individual. La parte correspondiente a la Escuela Técnica Superior de Ingenieros Aeronáuticos, el número de participantes que la han llevado a cabo también ha oscilado en el ámbito de 10 a 15 alumnos/as. En este caso, la práctica no se circunscribía en una asignatura especial sino que se les ofrecía al alumnado matriculado en la asignatura de "Idioma Técnico Moderno". En ambos casos, en segundo año de carrera.

Antes de entrar en materia, no vendría mal echar un vistazo a algunas palabras relacionadas con este tipo de actividad para comprobar su valor:

Practical letter writing is not only a social skill, it's a way of keeping the lines of communication between you and the others in good working order. And it's an effective way to stand up for your rights when dealing with companies, service people, ... Más tarde prosigue con una idea que creemos que nos concierne: your objective is to write letters that communicate rather than simply inform.(Baugh, 1991).

\section{Nivel de conocimientos.}

Con el fin de conocer con exactitud el nivel de conocimientos del alumnado participante en la práctica anteriormente citada, este fue consultado por medio de una encuesta, realizada en el curso 2001-2002, fruto de la cual se obtuvieron los datos que se exponen a continuación.

\section{Resultados de la encuesta.}

$\checkmark$ El $100 \%$ de los encuestados han redactado anteriormente textos en inglés. La extensión de los documentos elaborados, teniendo en cuenta que pueden trabajar con más de un tipo de texto, se reparte de la forma siguiente:

Textos de tipo medio (redacciones) 88,4\%

Textos cortos (resúmenes) $\quad 62,8 \%$

Textos largos (trabajos) $\quad 25,6 \%$

Otros (traducciones, etc.) $\quad 10,5 \%$

$\checkmark \quad$ El 92,7\% de los encuestados ha escrito anteriormente cartas en inglés. Los tipos de cartas más frecuentes son:

$\begin{array}{lr}\text { Dirigidas a amigos o familiares } & 55,8 \% \\ \text { Cartas académicas } & 37,2 \% \\ \text { Correo electrónico } & 32,5 \% \\ \text { Cartas laborales } & 11,6 \%\end{array}$

$\checkmark$ Tomando como muestra el grupo anterior aquellos que ya habían redactado cartas en inglés- el grado de éxito obtenido en su propósito inicial fue considerado por sus autores:
Bajo o medio
$67,5 \%$
Alto o muy alto
$32,5 \%$

Las causas del bajo resultado obtenido se atribuyen mayoritariamente al desconocimiento de las expresiones, a una preparación escolar insuficiente y a la poca experiencia. A distancia quedaron la falta de motivación y causas no debidas al idioma.

\section{Análisis de los resultados iniciales.}

De acuerdo con las respuestas obtenidas en el entorno académico del que proceden este alumnado (situación en el curso 2001/2002) podemos obtener las siguientes conclusiones, siempre teniendo en cuenta que estas son referidas a la evaluación inicial:

- Se trata de un alumnado que, según afirma, sabe escribir textos en inglés. Su formación se debe a la preparación para las pruebas de selectividad. 
- Es una formación basada en el inglés general. El planteamiento de la enseñanza de un segundo idioma en colegios e institutos no distingue orientaciones profesionales.

- Su nivel de preparación es medio, salvo excepciones. Todo el alumnado encuestado se encuentra en su segundo año de carrera y su nivel de inglés equivaldría al Primer Ciclo de inglés de la Escuela Oficial de Idiomas. En general podemos destacar su modestia al valorar sus conocimientos. Ello es debido a la dificultad para acceder a sus Escuelas y por otro lado, a que al responder a la encuesta, se encuentran iniciándose en el inglés para fines específicos (o inglés para entornos profesionales).

- No han redactado anteriormente cartas comerciales. Mayoritariamente han escrito cartas, pero casi siempre en ámbitos familiares o exentos de rigor formal.

En resumen, son alumnos que usan el inglés como herramienta de uso general. El ámbito que el profesorado de idiomas para entornos de ingeniería de la Universidad Politécnica de Madrid (UPM) crea en sus Escuelas es el de ser capaces de comunicarse en otro idioma (e incluso en el suyo propio). Y parte de esa comunicación es la de poder escribir cartas comerciales.

\section{2.- ANÁLISIS DE NECESIDADES PARA EL CONOCIMIENTO DEL GÉNERO DE LA CORRESPONDENCIA COMERCIAL Y PROFESIONAL EN INGLÉS.}

\section{Razones que derivan del marco académico.}

La carga lectiva del alumnado de ingeniería le impide conocer el mundo laboral, al que se incorpora una vez terminan la carrera. Esto, unido al hecho de que la tendencia actual en la universidad es la de comprimir el plan de estudios, no supone en absoluto una situación favorable a que el alumnado tenga un conocimiento más o menos exhaustivo de los requerimientos del mundo laboral.

La enseñanza de inglés para Entornos de Ingeniería en la Universidad Politécnica de Madrid, se inserta en este marco de un alumnado sometido a un gran esfuerzo de dedicación en un espacio de tiempo muy limitado.

El objetivo del profesorado de inglés para Entornos de Ingeniería ha de consistir, pues, en lograr que el alumnado obtenga la mayor rentabilidad en la ratio tiempo-esfuerzo.
Para superar estas limitaciones, creemos que el método de aprendizaje situacional es el que acerca más al alumnado al mundo real. Decidimos, por tanto, dedicar el tiempo con que contamos tanto profesorado como alumnado a plantear situaciones prácticas que le sitúen en un entorno lo más parecido posible al profesional, en el que se verá inmerso al finalizar sus estudios universitarios.

\section{Razones que derivan del mundo profesional.}

El principal objetivo de la universidad es preparar al futuro ingeniero (o licenciado) para que llegue al mundo laboral con todas las garantías de éxito posibles.

Gran parte de las empresas requieren que el ingeniero pueda comunicarse eficazmente con sus interlocutores, sean estos colaboradores, clientes, proveedores, etc. Para ilustrar esto, podemos echar un vistazo a lo que nos dice Rosset (1994):

Letter writing is of the utmost importance in the administration of any business, as the greater part of the world's transactions are carried out by means of letters. A letter is the connecting link between businessmen. Letters offer the medium through which companies get in touch; articles are offered for sale; prices are stated; purchases and sales are made; the terms of contracts are confirmed; payments are made; accounts are rendered and debts are recovered.

Podríamos afirmar por otro lado, que la labor de la secretaria tradicional se circunscribe a las altas esferas de dirección. La secretaria ha dejado de ser una persona auxiliar del ingeniero. Las tareas de redacción de cartas, memoranda, informes y otros documentos escritos, son ahora responsabilidad del propio ingeniero.

Dicho esto, nuestra labor docente implica que los materiales didácticos estén al día y utilizar el material auténtico lo más posible. La práctica que presentamos fue planteada a partir de situaciones reales en las que las empresas y clientes se intercambian anuncios, cartas y contratos. Nuestro alumnado las redacta con total fidelidad a esos modelos reales.

\section{3.- PRÁCTICA DE CORRESPONDENCIA COMERCIAL.}

Esta práctica va dirigida a los alumnos matriculados en segundo curso de carrera de 
Ingeniería Aeronáutica Superior y de Escuela Universitaria de Informática de la Universidad Politécnica de Madrid. La asignatura se denomina, Inglés Técnico II; desde un principio se reparten los papeles de proveedores y clientes, para lo cual los alumnos crean sus propias empresas. La comunicación se produce entre dichas empresas, no entre particulares. Previamente a la realización de la práctica los alumnos han de haber consultado y recopilado modelos de material adaptado y auténtico en la biblioteca de su unidad docente o sección departamental, además de la teoría impartida en clase. Los modelos de cartas e información al respecto que encuentran en Internet siempre resulta una valiosa ayuda (ver sitios Web recomendados).

La práctica consta de varias fases, cada una de las cuales se corresponde con una diferente función comunicativa. Existen limitaciones temporales para cada fase. Se establecen fechas límite para entregar las cartas por evidentes razones de operatividad.

\section{Objetivos.}

A continuación se detallan los objetivos didácticos y comerciales que se desean alcanzar:

$1^{a}$ Fase. Redacción de una carta comercial dirigida a un potencial cliente. Se intenta fomentar la creatividad del alumno, que inventa su propia empresa y logotipo. Los proveedores (alumnado de la Escuela emisora) se anuncian en cartas abiertas. La información debe aparecer planteada del modo más conciso y atractivo posible a fin de captar la atención del destinatario. Todos ofrecen los mismos productos o servicios a los mismos clientes (el alumnado participante en la Escuela receptora). Este paso es crucial, pues de la primera impresión que cause la empresa depende en gran medida su posterior éxito o fracaso. Los anunciantes deben hacer mención inicial de precios de sus productos en esta primera fase.

$2^{a}$ Fase. Petición de información, detalles y pormenores del producto. Los clientes seleccionan los tres mejores anuncios según su criterio y responden mostrando su interés inicial. Solicitan información detallada sobre los bienes, precios o servicios ofertados.

$3^{a}$ Fase. Descripción de los productos o servicios. Los proveedores inician su oferta al potencial cliente enviándole una información exhaustiva sobre sus productos. Se puede iniciar un debate sobre el precio inicial. Es importante resaltar que todos los proveedores siempre parten con unos mismos precios de coste de sus bienes o servicios. Deberán operar siempre por encima de este margen pues, de lo contrario, entrarían en pérdidas pese a obtener más clientes. Ésta no es una opción aceptable. Aquí el alumno deberá reflexionar en función de su situación sobre si le interesa más a su empresa tener muchos clientes con una menor ratio beneficio-cliente o si, por el contrario, puede permitirse tener pocos clientes que le proporcionen unos ingresos elevados.

$4^{a}$ Fase. Negociación. Contraste de pareceres. Los clientes tratan de obtener mejores condiciones, ya sea en precios o prestaciones. Planteamiento de contraofertas.

$5^{a} \quad$ Fase. Condiciones definitivas. Descripción de condiciones definitivas. Los proveedores contestan a sus clientes tratando de obtener un acuerdo que satisfaga a ambas partes. Se envía una propuesta definitiva.

$6^{a}$ Fase. Pedidos. Aceptación de condiciones. Los clientes eligen la mejor opción de entre las ofertas recibidas y hacen un pedido formal.

$7^{a}$ Fase. Redacción de un contrato. Documentación sobre facturas y contratos. Los proveedores envían una factura o contrato para que los clientes lo firmen.

$8^{a}$ Fase. Firma del contrato. Los clientes leen el contrato y lo firman. La firma de contratos es la meta última de los proveedores y la medida fiable de su grado de éxito.

Toda la correspondencia está canalizada por el profesorado responsable de la práctica en cada centro. Se conservan los originales creados por el alumnado y se envían copias para su distribución en el centro receptor. Esta norma se puede alterar en la primera etapa, en la cual se envían los originales y se conservan las copias. Esto se hace con aquellas cartas cuyo especial diseño perdería atractivo en caso de ser fotocopiadas.

\section{Calificación de la práctica.}

Una vez finalizado el plazo establecido se procederá a evaluar la práctica de la siguiente manera:

- Cada profesor evaluará la correspondencia de sus propios alumnos.

- La evaluación seguirá un procedimiento diferente según se esté calificando a las empresas vendedoras o a las empresas que actúan como clientes. 


\section{Vendedores}

Los vendedores parten de una situación de competencia real entre ellos, puesto que han de ofertar los mismos productos y servicios a los mismos clientes.

En el caso de los vendedores la calificación final de la práctica será la que se obtenga de hacer la media de las notas en los apartados de lingüística y comercial. Esto se hace para incentivar más al alumno: el que haya una clasificación final añade un ingrediente de competencia comercial muy valorado por los participantes.

\section{Calificación}

A continuación se detalla el procedimiento de calificación de cada apartado.

Para la calificación comercial se establecerá un ranking en función de los resultados económicos obtenidos tras descontar los costes iniciales que, como se ha mencionado anteriormente, son prefijados e iguales para todas las empresas.

- No firmar contrato alguno implica suspender este apartado.

- El haber firmado al menos un contrato supone el aprobado (5).

- La nota máxima (10) la obtendrá la empresa que más beneficios obtenga.

- Las notas restantes -desde 5,x hasta 9,xse reparten de acuerdo con una estimación ponderada en función de los beneficios de cada empresa.

El siguiente caso práctico puede servir de aclaración:

Supongamos que, en un conjunto de 8 empresas se obtuvieran los siguientes resultados:

La empresa A obtiene $\quad 125.000 €$

Las empresas B y C $\quad 115.000 €$

La empresa D $\quad 85.000 €$

Las empresas E y F $\quad 75.000 €$

La empresa $G \quad 65.000 €$

La empresa $\mathrm{H} \quad 25.000 €$ (sólo un contrato)

Las calificaciones del apartado comercial de estas empresas serían las siguientes:

$\begin{array}{ll}\text { Empresa A } & 10 \text { puntos } \\ \text { Empresas B y C } & 9,5 \\ \text { Empresa D } & 8 \\ \text { Empresas E y F } & 7,5\end{array}$

$\begin{array}{ll}\text { Empresa G } & 7 \\ \text { Empresa } \mathrm{H} & 5\end{array}$

Como se puede apreciar, el estar comprendido el máximo (10) y el mínimo (5) entre $125.000 €$ y $25.000 €$ hace que cada $10.000 €$ entre estos márgenes equivalgan a 0,5 puntos en la calificación final.

Esta evaluación comercial se realizará preferentemente ante todos los componentes de las empresas, ubicando a cada una en un panel o cualquier otro lugar donde se pueda visualizar fácilmente la respuesta obtenida en cada una de las comunicaciones, descubriendo los alumnos de este modo las razones de su clasificación.

La calificación lingüística se obtendrá restando puntos de un total inicial de 100 -del que parten todos los grupos/empresas-. Estos puntos se descuentan por los conceptos desarrollados según el siguiente baremo:

Gramática:

$40 \%$

Estilo: $30 \%$

Vocabulario: $15 \%$

Distribución y presentación: $15 \%$

En caso de no haber cometido ningún error, la calificación final sería de 10.

Por ejemplo: si un alumno o alumna obtiene un $25 \%, 15 \%, 8 \%$ y $6 \%$ en cada uno de los apartados, su calificación final será de $54 \%$, es decir, de 5,4.

Con este sistema se pretende motivar al alumnado a participar en la práctica, pues en muchas ocasiones esos puntos les sirven para alcanzar un nivel superior de calificación, ya sea de aprobado a notable o de notable a sobresaliente, dependiendo de cada caso.

Aparte de esto, el alumnado puede acudir a tutorías durante todo el proceso para aclarar las dudas que pudieran surgir en cuanto a la redacción de las cartas.

\section{Compradores}

El objetivo de los compradores es, obviamente, realizar una compra de bienes o servicios con la mayor rentabilidad posible para su empresa. Han de realizar una labor de selección y evaluación a lo largo de la práctica consistente en saber escoger la empresa vendedora que le dé mejor producto y al mejor precio.

\section{Calificación}

En este caso se establecen unos parámetros de calificación que responden a los siguientes apartados y ratios: 
Gramática:

$20 \%$

Estilo:

$20 \%$

Vocabulario:

$10 \%$

Distribución y presentación:

$10 \%$

Grado de éxito en la finalización

$40 \%$

La fórmula de corrección es sencilla: con las cartas de todo el proceso se otorga al alumnado un $100 \%$ inicial, del que se van quitando décimas según se van encontrando errores definidos en el anterior baremo. El resultado final será una nota de 1 punto si no se han cometido errores o las décimas de punto que queden tras la resta de errores en el cómputo global.

\section{Dificultades encontradas en la realización de la práctica.}

La principal dificultad con que nos topamos al afrontar la práctica es el total desconocimiento por parte del alumnado del género epistolar comercial. La simulación se desarrolla en paralelo con el cuatrimestre y, sobre todo en las primeras semanas, el alumnado descubre que sus destrezas comunicativas son realmente limitadas, y no sólo en inglés, sino fundamentalmente y como razón principal en su lengua materna, el español. La escasa capacidad de expresión escrita que caracteriza a la mayoría del alumnado de ingenierías es el principal escollo que debemos salvar.

El proceso comienza por enseñarle a estructurar sus ideas de forma lógica para luego poder formularlas por escrito con claridad y coherencia. Podríamos afirmar que domina más los números que las letras. Son alumnos acostumbrados a resolver problemas lógicos, a pensar, pero no tanto a plasmar los resultados de esos pensamientos en frases sencillas $y$ comprensibles.

Cuando hemos logrado que se expresen con claridad en español podemos dar el paso al segundo idioma. El trabajo tutorial es por lo tanto imprescindible en estas primeras semanas y esta constante supervisión hace que la práctica no resulte viable en caso de trabajar con grupos muy numerosos.

\section{Análisis de errores más comunes por apartados.}

Las aplicaciones de proceso de texto actuales (fundamentalmente Microsoft Word y otros) ofrecen entre sus opciones de menú, la posibilidad de acceder a plantillas de múltiples documentos entre los que se encuentran modelos de cartas comerciales.

Nuestra recomendación a los alumnos, que en su práctica totalidad son usuarios de algún tipo de ordenador personal, es que sigan las pautas marcadas por estos modelos.

A continuación plantearemos los errores más comunes cometidos por los alumnos participantes en las sucesivas ediciones de la práctica, agrupados por apartados según el esquema de una carta.

\section{En el encabezamiento}

Algunos de los errores más frecuentes cometidos por los alumnos son los siguientes:

Datos incompletos.

- Ubicación incorrecta de datos.

- Ausencia de algún elemento del apartado (remitente, fecha o destinatario).

- Orden incorrecto de los datos (por ejemplo: cargo antes del nombre).

- Formato inadecuado (por ejemplo: no respetar márgenes o distancias).

- Errores de tipo ortográfico.

En fórmulas de salutación, despedida y firma.

$\square$ En fórmulas de salutación:

1.- Dear Sir Smith. Uso de Sir en lugar de $M r$., frecuente en español por la similitud de Sir y Señor.

2.- DearMr John Smith. No es correcto escribir el nombre propio del destinatario detrás del trato de cortesía. Mr. y Ms. sólo han de ir seguidos por el apellido. La fórmula Ms. hace referencia tanto a mujeres casadas (Mrs.) como solteras (Miss) Estas fórmulas que indican estado civil se utilizarán cuando así lo hayan indicado con su uso previo las propias personas mencionadas.

3.- DearTechnical Director. La fórmula de cortesía Dear sólo admite dos posiblidades:

- Seguida de un nombre propio, si la relación es de familiaridad: Dear John:

- Seguida de $\mathrm{Mr} / \mathrm{Ms}+$ apellido, como fórmula general: Dear Mr. Smith:

4.- Dear John Smith. Como se menciona en el caso anterior. Dear no suele ir seguido de nombre y apellido del destinatario. 
5.- Ausencia de salutación. Incluso si no sabemos quién va a leer la carta y, por tanto, a qué persona dirigirla, debemos incluir una fórmula de salutación. En estos casos podemos optar por las soluciones siguientes:

- Dear Sir/Madam:

- To whom it may concern: (a quien corresponda)

- Gentlemen/ Mesdames (en Estados Unidos)

6.-For the attention of John Smith. En este caso el error es doble puesto que, por una parte, for the attention of debe ir en el lugar correspondiente del encabezamiento de la carta junto con los datos del destinatario. Además, se ha omitido el trato de cortesía Mr.

7.- Marketing Manager: No debemos saludar al destinatario refiriéndonos a él por su cargo, sino siempre por su nombre, según se indica en los puntos anteriores.

8.- Mr Smith: La omisión de la fórmula de cortesía Dear confiere al saludo una rudeza que sólo sería comprensible en una carta de queja contundente. Incluso en este tipo de carta es recomendable no salirse de las fórmulas de cortesía habituales. La dureza debe estar en el contenido, en las razones que se aportan en el cuerpo de la carta, pero no en la forma. La razón no está reñida con los buenos modales. (Manners maketh man).

9.- Uso de signos de puntuación incorrectos tras la salutación. Lo habitual es poner coma o dos puntos detrás del saludo de cortesía. También es correcto no poner nada, omitir el signo de puntuación. En ningún caso se debe utilizar punto, o punto y coma.

$\square$ En fórmulas de despedida:

Si hemos comenzado por Dear Sir/Madam, es decir, si no conocemos el nombre del destinatario, lo correcto es finalizar con Yours faithfully. (En Estados Unidos las cartas iniciadas con la salutación Gentlemen/Mesdames finalizan habitualmente con Yours truly).

En los demás casos utilizaremos Yours sincerely. Usar únicamente Yours o Best wishes implica una gran familiaridad.

Son también formulas de cortesía habituales para las despedidas:

- Looking forward to hearing from you, o

- I/We look forward to hearing from you soon/in short
El uso de estas expresiones no supone omitir el cierre mencionado anteriormente, Yours faithfully, para destinatarios desconocidos o Yours sincerely en los demás casos. (Yours truly en EEUU). Suelen situarse un espacio por debajo de la última línea del cuerpo de la carta.

La despedida se escribirá a uno o dos espacios de la última línea.

La puntuación posterior a la expresión de despedida sigue los mismos criterios que aplicábamos a la salutación y suele ser coherente con ésta. Si no hemos incluido puntuación alguna tras el saludo, no lo haremos tampoco ahora; si pusimos dos puntos tras el saludo, también lo haremos ahora, y si pusimos una coma, volveremos a hacerlo.

Algunos errores comunes en las fórmulas de despedida son:

Looking forward to hear from you.En esta expresión, el uso del infinitivo to hear es inaceptable, pese a que pudiera parecer gramaticalmente correcto. Se trata de fórmulas de cortesía que no se pueden alterar. Debería ser to hearing.

Comenzar Dear Sir y terminar con Yours sincerely o comenzar Dear Mr. Smith y terminar con Yours faithfully. Debe haber coherencia en el uso de las fórmulas de salutación y despedida.

Omitir la despedida. Resulta inexcusable y es una falta de cortesía. De nuevo hay que insistir en que las fórmulas de cortesía son independientes de nuestro estado de ánimo y del contenido de la carta.

$\square$ En la firma:

En cuanto a la firma de las cartas, los errores más comunes son:

1.- No firmar la carta.

2.- Firmar en una posición centrada. (admisible sólo por razones de estética).

3.- Omitir el nombre de quien firma o el puesto que ocupa en la empresa.

4.- Añadir postdatas tras la firma. Son propias de correspondencia más informal o personal. En correspondencia comercial se supone que las cartas han sido meditadas y analizadas detalladamente con anterioridad a su redacción. No queda, pues, sitio para la improvisación y el olvido que son la razón de ser de las postdatas. 
En el cuerpo de la carta

\section{$\square$ Errores gramaticales}

We have been studing differents programmes.-El verbo study en gerundio precisa una $y$ (studying). Por otra parte, el adjetivo differents recibe una $s$ en el plural que no debiera figurar pues los adjetivos nunca se pluralizan.

Will give free updatesduring 3 years.- el uso de during y for sigue sin estar muy claro entre el alumnado. En este caso debe ser: for 3 years. El uso de during se limita a la necesidad de mencionar el tiempo de que se trate, por ejemplo: during the summer, during your stay in our company, etc.

We also would like to could offer you the best service.-El alumno quería decir: también nos gustaría poder ofrecerle. En este caso, ha añadido un auxiliar modal tras la partícula to. La expresión poder ofrecerle sería mejor decirla: to be able to offer you.

It would also be interesting to know how many hours does it need to be installed.-En este caso, el error se produce al introducir un auxiliar does en una frase donde no existe tal posibilidad al no tratarse ni de un auxiliar enfático ni una pregunta. Eliminarlo sería la opción correcta: to know how many hours it needs to be installed. .

$\square$ Errores de presentación, estilo, composición, exposición y distribución.

Expecting your company will send us.Este comienzo suena demasiado a una mala traducción del español.

We wait for your final contract.- Otro uso del verbo esperar erróneo. El presente simple se utiliza en acciones de frecuencia, cual no es el caso. La frase más correcta sería: we are looking forward to receiving the contract.

En ciertas cartas, el alumno no hace separación de una línea o más entre párrafos, lo que hace que la lectura se haga a través de un texto demasiado compacto para la vista. Este error de distribución de párrafos se subsanaría sencillamente separando los párrafos con una línea en blanco. Si la carta fuera demasiado larga, se deberían añadir estas líneas, disminuyendo además el tamaño de la letra.

Hoping to hear from you soon.- El alumno o alumna ha traducido literalmente: esperando tener noticias.... Podría decirse: we hope to hear.
In your letter, you did not mention any exhibition, we take a special interest in the demonstration.-Se usan comas que lejos de aclarar la idea de la frase, entorpece su comprensión. Por otro lado, no hay cohesión en la frase, pues la segunda parte no parece corresponderse con la primera. Proponemos como solución: you did not mention any presentation of the product in your letter, and we are very interested in one.

\section{$\square$ Errores léxicos y ortográficos}

In the informatic world.- La informática es una ciencia cuya traducción al inglés es: computer science. En este caso, sencillamente computer world.

We thank you the letter.-Debe incluirse una preposición for antes del objeto directo, y en todo caso, sugerimos el uso de appreciate en este caso concreto: we appreciate your letter.

... and arriving at the conclusion.- No se debe usar la expresión arrive at cuando nos referimos a llegar a la conclusión. En este caso debe expresarse: get to the conclusion o sencillamente: and concluding that...

Your possible buying.-No es una buena elección el verbo buying. Purchase es una buena opción en este caso, palabra muy extendida en el mundo de los negocios. Confiere el sentido de adquisición que damos a las cosas valiosas.

\section{4.- CONCLUSIÓN Y PERSPECTIVAS FUTURAS.}

Antes de finalizar nos gustaría incluir algunas palabras que consideramos muy ilustrativas al respecto de esta práctica, a modo de resumen:

Correspondence is essential in establishing and confirming transactions in commerce. Typed or produced on a word processor it reflects you or your business. Therefore what is written and how it is expressed is as much a part of a business education as accountancy and economics. (Ashley, 1992).

Con este aprendizaje práctico el alumno comprueba que al tiempo que adquiere nuevos conocimientos ve que el trabajo que realiza puede serle de gran utilidad en el futuro. A su vez permite al docente detectar y ayudar a corregir los errores que con mayor frecuencia comete el estudiante al escribir correspondencia comercial en inglés.

Por último, los profesores que hemos llevado a cabo esta experiencia pensamos que esta 
práctica podría abrir un espectro de posibilidades a docentes cuyo interés incluya la enseñanza y práctica de diversas destrezas comunicativas tales como entrevistas de trabajo, conversaciones telefónicas, presentaciones de diversa índole, reuniones, negociaciones y cualquier otro tipo de situaciones profesionales para las que el alumno debe estar lo más preparado posible una vez se incorpore al mundo laboral.

\section{5.- REFERENCIAS BIBLIOGRÁFICAS.}

Ashley, A. (1992). A Handbook of Commercial Correspondence. Oxford. Oxford University Press.

Baugh, L.S. (1991). Handbook for Practical Letter Writing. Lincolnwood, Illinois. NTC Publishing Group.

Rosset, E. R. (1994). Bilingual Business Letters. Cartas comerciales bilingües. Irún: Stanley.

\section{ANEXO}

\section{Sitios Web Internet recomendados:}

http://www.everythingemail.net/email_help_tips. html

http://www.letters-4u.com/letters

http://www.plainenglish.co.uk/letterguide.html http://www.webfoot.com/advice/email.top.html http://www.businesstown.com

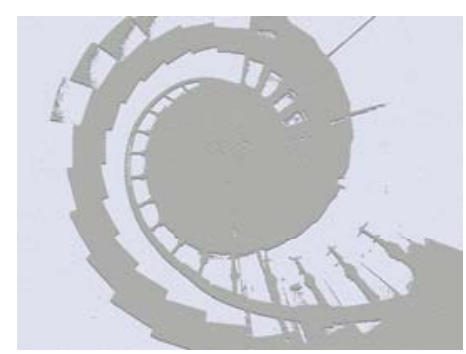

